\title{
Stability criterion for attractive Bose-Einstein condensates
}

\author{
Luc Bergé ${ }^{1}$, Tristram J. Alexander ${ }^{2}$, and Yuri S. Kivshar ${ }^{2}$ \\ ${ }^{1}$ Commissariat à l'Energie Atomique, CEA/Bruyères-le-Châtel, B.P.12, 91680 Bruyères-le-Châtel, France \\ ${ }^{2}$ Optical Sciences Center, Australian National University, Canberra ACT 0200, Australia
}

\begin{abstract}
A general stability criterion is derived for the ground states of the Gross-Pitaevskii equation, which describes attractive Bose-Einstein condensates confined in a magnetic trap. These ground states are shown to avoid the collapse in finite time and are proven to be stable in two and three spatial dimensions.
\end{abstract}

PACS numbers : 03.75.Fi, 03.65.Ge, 05.30.Jp, 47.20.Ky, 02.30.Jr

\section{INTRODUCTION}

Experimental observation of Bose-Einstein condensation (BEC) in ultracold atomic clouds [1] has stimulated a new direction in the study of macroscopic quantum phenomena. Basically, the interaction between two confined bosons in a condensate is determined by the s-wave scattering length $a_{0}$ and it can be either repulsive $\left(a_{0}>0\right)$ or attractive $\left(a_{0}<0\right)$. Although first BEC experiments were commonly realized with gases promoting a positive scattering length, trapped ${ }^{7} \mathrm{Li}$ atom gases, which are characterized by a negative scattering length, have raised an increasing interest 2 justified by the rich and complex dynamics mixing instability and generation of solitonlike structures, which substantially alters the formation of condensates. Furthermore, novel experimental results [3] suggested the possibility of using so-called Feshbach resonances to continuously detune $a_{0}$ from positive to negative values by means of an external magnetic field, which brings a new insight into the experimental realization of BECs with attractive interactions.

The dynamical behaviors of gases with negative scattering length are especially interesting, because in the absence of trapping the condensate is described by the nonlinear Schrödinger (NLS) equation, whose localized, multi-dimensional solutions are unstable and may col-

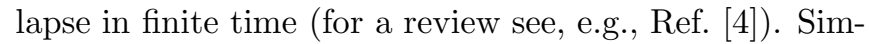
ilarly, collapse also occurs in confined condensates with attractive interactions, as emphasized by many theoretical works [5]7], and sequences of collapse events have been experimentally detected in BECs of ${ }^{7} \mathrm{Li}$ gas [8], in which the condensate was observed to shrink on time scales of the trap oscillation period. General conditions for collapse follow from the virial theorem applied to the Gross-Pitaevskii (GP) equation

$$
i \hbar \frac{\partial \psi}{\partial t}=-\frac{\hbar^{2}}{2 m} \nabla^{2} \psi+\frac{m \omega^{2}}{2}\left(r^{2}+\gamma^{2} z^{2}\right) \psi+U_{0}|\psi|^{2} \psi,
$$

where $\psi$ denotes the BEC wavefunction, $\nabla^{2}$ is the threedimensional Laplacian, $\gamma$ measures the anisotropy ratio of the condensate and $U_{0} \equiv 4 \pi \hbar^{2} a_{0} / m$ characterizes the two-body interaction ( $m$ is the atom mass and $\omega$ the trap frequency). These conditions imply the existence of a critical value, $N_{\text {cr }}$, that the total number of atoms $N \equiv \int|\psi|^{2} d \vec{r}$ must exceed for initiating the collapse. For $N$ below critical, attractive condensates are now known to be capable of relaxing to stationary states of the GP equation, reading as $\psi(\vec{r}, t)=\phi(\vec{r}) \exp (-i \mu t / \hbar)$, where $\mu$ has the meaning of a chemical potential. These solutions consist of solitonlike ground states, whose stability was recently analyzed by means of numerical continuation methods [9]. It was in particular revealed that as $N$ approaches $N_{\text {cr }}$ from below, the condensate modeled by this ground state becomes more unstable and no stationary condensate forms for $N>N_{\text {cr. }}$. Besides, a new investigation [10] showed that BEC with large negative scattering length can be modulationally unstable and decay into periodic fringe patterns of parallel solitons, which again stresses the important role of the latter in this field.

In spite of these recent results, some fundamental points, however, remain unsolved. First, conditions for the existence of stationary condensates have never been established for high-dimensional systems and we still do not know how they may be compatible with the conditions for collapse, which mainly follow from the Hamiltonian properties of the GP equation. Second, we ignore how trapped condensates can evolve towards ground states. Third, for practical uses, it is highly desirable to know whether stability of stationary condensates can be inferred from their number of particles, and from this number only. The aim of this paper is to answer these questions and to clear up the link between the non-occurrence of collapse with respect to the stability of localized, stationary ground states when $a_{0}<0$. In the following, we review the conditions required for the collapse of two-dimensional (2D) and three-dimensional (3D) condensates and present a rigorous derivation of the stability criterion for the ground-state solutions of the GP equation. 3D isotropic BECs are analyzed in the limit $\gamma^{2} \rightarrow 1$, for which radially-symmetric solutions only depend on the radius $r=\sqrt{x^{2}+y^{2}+z^{2}}$ in 
Eq. (1). In 2D, the model (1) can be employed to describe the dynamics of anisotropic condensates having a pancake-shape geometry with $\gamma^{2} \gg 1$. In this configuration, $\psi=\psi^{\prime}(x, y, t) \phi(z)$ exhibits a longitudinal component, $\phi(z)$, frozen on the Gaussian shape $\phi(z)=(\gamma / \pi)^{1 / 4} \exp \left(-\gamma z^{2} / 2\right)$ 10,11. The condensate dynamics is then basically governed by the $2 \mathrm{D}$ version of Eq. (11) with radius $r=\sqrt{x^{2}+y^{2}}$. 1D BECs are disregarded, since related results are already available in the current literature devoted to optical solitons [12]. Instead, we focus our attention on situations allowing for collapse, which basically concerns high dimension numbers [13]. As collapse properties depend on more than the dimensionality rather than the geometry of nonlinear objects, we only address the stability of isotropic condensates.

\section{COLLAPSE VS. GROUND STATES}

¿From the viewpoint of the nonlinear dynamics of localized modes, studying multi-dimensional trapped condensates is equivalent to investigating the solutions of the $D$-dimensional equation

$$
i \partial_{t} u+\nabla^{2} u-\frac{\Omega_{0}^{2}}{4} r^{2} u+|u|^{2} u=0,
$$

where $\partial_{t}$ is a partial derivative in time and $\nabla^{2} \equiv$ $r^{1-D} \partial_{r} r^{D-1} \partial_{r}$. In Eq. (2), the time and space variables are measured in units $(\hbar / m \omega)^{1 / 2}$ and $2 / \omega$, respectively; the new wavefunction $u$ scales as $\left(\hbar \omega / 2 U_{0}\right)^{-1 / 2}$, and $\Omega_{0}=2$ stands for the trap frequency $\omega$, which is formally kept undefined for technical convenience. Briefly speaking, collapse occurs as the mean-square radius of the wavefunction, $\left\langle r^{2}\right\rangle \equiv(1 / N) \int r^{2}|u|^{2} d \vec{r}$, where $N=$ $\int|u|^{2} d \vec{r}$ accounts for the rescaled particle number, tends to zero in finite time, while the amplitude of $u(\vec{r}, t)$ diverges in the same limit. To be triggered off, this singular phenomenon requires special conditions on the initial data $\left.R_{0}^{2} \equiv\left\langle r^{2}\right\rangle\right|_{t=0}$ and $\left.\dot{R}_{0}^{2} \equiv \partial_{t}\left\langle r^{2}\right\rangle\right|_{t=0}$, among which we henceforth consider wavefunctions with no initial divergence and set $\dot{R}_{0}=0$, for the sake of clarity. These requirements are basically inferred from the socalled "virial" relation

$$
\partial_{t}^{2}\left\langle r^{2}\right\rangle=\frac{8 H}{N}+\frac{(4-2 D)}{N} \int|u|^{4} d \vec{r}-4 \Omega_{0}^{2}\left\langle r^{2}\right\rangle,
$$

which is merely derived by multiplying Eq.(2) with $\left(r^{2} u^{*}\right)$ and $\left(\vec{r} \cdot \nabla u^{*}\right)\left({ }^{*}\right.$ means complex conjugate $)$ and by combining the imaginary and real parts of the results, respectively [5 6,13]. Equation (3) then shows that the meansquare radius $\left\langle r^{2}(t)\right\rangle$ inevitably vanishes at a finite time, $t_{c}$, whenever $D \geq 2$ and when the conserved Hamiltonian integral

$$
H \equiv X-Y+\frac{\Omega_{0}^{2}}{4} N\left\langle r^{2}\right\rangle,
$$

where

$$
X \equiv \int|\nabla u|^{2} d \vec{r}, \quad Y \equiv \frac{1}{2} \int|u|^{4} d \vec{r}
$$

fulfils conditions making the right-hand side of (3) negative.

Two analytical estimates are usually employed to determine necessary requirements on the particle number for initiating the collapse [14,15], namely, the "uncertainty" inequality

$$
N \leq(2 / D)^{2} X\left\langle r^{2}\right\rangle,
$$

from which the gradient norm $X$ blows up as $\left\langle r^{2}\right\rangle \rightarrow 0$, and the Sobolev inequality

$$
Y \leq\left(2 / D N_{0}\right)(4 / D-1)^{D / 2-1} X^{D / 2} N^{2-D / 2} .
$$

This expression proceeds from optimizing the estimate $Y \leq C X^{D / 2} N^{2-D / 2}$ by means of variational methods, which amount to minimizing the functional $J\{u\}=$ $X^{D / 2} N^{2-D / 2} / Y$ and provide a dependence between the best constant $C_{\text {best }}$ and the quantity $N_{0}$. Here, $N_{0}$ is the value of $N$ computed with the radially-symmetric ground-state $\chi_{0}(r)$ satisfying the NLS equation $-\chi_{0}+$ $\nabla^{2} \chi_{0}+\chi_{0}^{3}=0$ with no trap, such that $N_{0}=11.68$ for $D=2$ and $N_{0}=18.94$ for $D=3$ [14]. When $\Omega_{0}=0$ [free NLS], a sufficient condition for collapse is $H \leq 0$. In the 2D case, Eq. (7) then imposes that $N$ must be larger than $N_{\text {cr }}=N_{0} \simeq 11.7$. In the $3 \mathrm{D}$ case, sharper estimations of the complete identity (3), $\partial_{t}^{2}\left\langle r^{2}\right\rangle=(4 / N)(3 H-X)$, yield the more stringent sufficient condition for collapse $H<N_{0}^{2} / N$, after bounding $H$ from below with (7) [15]. 13.

When $\Omega_{0} \neq 0$, the mean-square radius $\left\langle r^{2}\right\rangle$ satisfies

$$
\left\langle r^{2}\right\rangle \leq\left(R_{0}^{2}-\frac{2 H}{N \Omega_{0}^{2}}\right) \cos \left(2 \Omega_{0} t\right)+\frac{2 H}{N \Omega_{0}^{2}},
$$

where the strict equality concerns the $2 \mathrm{D}$ case only, and $H \leq 0$ is also sufficient for collapse, since it leads to $\partial_{t}^{2}\left\langle r^{2}\right\rangle<0$ from Eq. (3). Because $H$ expands as $H=H_{\Omega_{0}=0}+H_{\text {cr }}$, where $H_{\text {cr }} \equiv \frac{1}{4} N \Omega_{0}^{2} R_{0}^{2}$, this requirement implies $H_{\Omega_{0}=0}<0$. For $D=2, N$ must thus necessarily exceed $N_{\mathrm{cr}}=N_{0}$ [5, 13]. In the opposite case $H>0$, two distinct dynamics can develop: (a) When $H_{\Omega_{0}=0}<0$, collapse occurs again within the domain $H<H_{\text {cr }}$ for which the existence of a finite maximal blow-up time, $t_{c}^{\max }=\left(1 / 2 \Omega_{0}\right) \cos ^{-1}\left[1 /\left(1-2 H_{\mathrm{cr}} / H\right)\right]$, makes sense. In the special case $N=N_{\text {cr }}\left(H=H_{\text {cr }}\right)$, $2 \mathrm{D}$ wavefunctions blow up with an amplitude diverging at $t_{c}=\pi / 2 \Omega_{0}$. (b) When $H_{\Omega_{0}=0}>0$ with $N<N_{\text {cr }}$, there is 
no collapse for $D=2$ : condensates having $H>H_{\text {cr }}$ never blow up and evolve by oscillating with the constant frequency $2 \Omega_{0}[16]$. For $D=3$, the latter conclusion must, however, be subdued, because sharper criteria for blowup may still apply [5, 15], so that $H>H_{\text {cr }}$ just consists of a necessary condition for the absence of collapse.

Hence, although reinforced with a parabolic trap [13], the collapse generally manifests under specific conditions, such as $H \leq H_{\text {cr }}=\frac{1}{4} N \Omega_{0}^{2} R_{0}^{2}$. In the opposite case, $H>H_{\mathrm{cr}}$, collapse can disappear and the wavefunction $u$ does not systematically spread out, but may instead oscillate or even form robust solitonlike states. This possibility of forming stable condensates remains an open problem for high dimension numbers. Therefore, it is worth knowing whether the stationary bound states of Eq. (2), expressing as

$$
u(\vec{r}, t)=\chi(\vec{r}) \exp (i \Lambda t)
$$

can exist for spatial dimensions $D \geq 2$. These ground states are defined by the solutions of the differential equation

$$
-\Lambda \chi+\nabla^{2} \chi-\frac{\Omega_{0}^{2}}{4} r^{2} \chi+|\chi|^{2} \chi=0
$$

which functionally depend on $\Lambda$ and are localized in space, i.e., $\chi(\vec{r})=0$ at $r \rightarrow+\infty$. Here, the parameter $\Lambda$ is sign-opposite to the energy eigenvalue $E=-\Lambda$, which corresponds to the above-defined chemical potential $\mu$ in reduced units. To start with, we examine the precise conditions under which these states may form. We multiply Eq. (10) by $\chi^{*}$ and $\vec{r} \cdot \vec{\nabla} \chi^{*}$, then combine the results to find

$$
\begin{aligned}
& X_{s}=\frac{N_{s}}{(4-D)}\left[D \Lambda+\frac{\Omega_{0}^{2}}{4}(D+4)\left\langle r^{2}\right\rangle_{s}\right], \\
& Y_{s}=\frac{N_{s}}{(4-D)}\left[2 \Lambda+\Omega_{0}^{2}\left\langle r^{2}\right\rangle_{s}\right],
\end{aligned}
$$

where subscript $s$ applies to the integrals computed on the ground-state solution. As $X_{s}$ and $Y_{s}$ are both positive quantities, it is easy to check that localized solutions $\chi$ exist for $D<4$, provided that

$$
\Lambda>-\frac{\Omega_{0}^{2}}{2}\left\langle r^{2}\right\rangle_{s}
$$

Unlike their free NLS counterparts $\left(\Omega_{0}=0\right)$, confined stationary states can thus exist for negative parameters $\Lambda$ and the requirement (12) implies that the energy $E$ must be less than twice the mean-square radius of the stationary condensates. Furthermore, the integral $H$ for the ground states reads

$$
H_{s}=\frac{D-2}{4-D} \Lambda N_{s}+\frac{\Omega_{0}^{2}}{4-D} N_{s}\left\langle r^{2}\right\rangle_{s},
$$

which ensures $\partial_{t}^{2}\left\langle r^{2}\right\rangle_{s}=0$ from Eq. (3), as expected. Combining relation (13) with the existence condition (12) then yields the constraint that the Hamiltonian must satisfy on the stationary solutions:

$$
H_{s} \geq \frac{\Omega_{0}^{2}}{2} N_{s}\left\langle r^{2}\right\rangle_{s}>0
$$

Thus, $H_{s}$ for steady solutions of trapped BECs is strictly positive. Moreover, we can observe from the result (14) that $H_{s}$ belongs to the range $H_{s}>\frac{1}{4} N \Omega_{0}^{2}\left\langle r^{2}\right\rangle_{s}$, where there is no collapse for $D=2$ if $N_{s}<N_{\mathrm{cr}}$, and where collapse can be prevented for $D=3$. On the other hand, since collapse implies the blow-up of the gradient norm $X \rightarrow+\infty$ as $\left\langle r^{2}\right\rangle \rightarrow 0$ [see Eq.(6)], a sharper condition assuring the collapse at dimensions $D>2$ can be inferred in the domain $X>X_{s}$ from the virial relation (3) rewritten as

$$
\partial_{t}^{2}\left\langle r^{2}\right\rangle=\frac{4 D}{N}[H-(1-2 / D) X]-(D+2) \Omega_{0}^{2}\left\langle r^{2}\right\rangle,
$$

and it reads $H \leq(1-2 / D) X_{s}$. From expression (13), $H_{s}$ can be seen to never belong to this class of Hamiltonians. Therefore, as $H_{s}$ always lies above this bound together with the limit value $H_{\text {cr }} \equiv \frac{1}{4} N \Omega_{0}^{2} R_{0}^{2}$ below which any initial datum certainly promotes the collapse, it is important to investigate the stability of the stationary states $\chi$.

\section{STABILITY CRITERION}

We first use the heuristic argument, following which the functional dependences of $H$ on $u$ under the constraint of a fixed particle number $N$ provides some qualitative information about stability. Indeed, as is readily seen from the variational problem $\delta(H+\Lambda N)=0, \chi$ governed by (10) realizes an extremum of $H$ at fixed $N$. Under this constraint, we employ the compatible substitution $u=a^{-D / 2} \chi(r / a)$, where $a$ plays the role of a Lagrange multiplier, and we plug it into $H$ to obtain

$$
H_{a}=X_{s} / a^{2}-Y_{s} / a^{D}+a^{2} I_{3}
$$

where the integrals $X_{s}$ and $Y_{s}$ are here expressed in terms of $\xi=r / a$, while $I_{3} \equiv \frac{1}{4} \Omega_{0}^{2} \int \xi^{2}|\chi(\xi)|^{2} d \vec{\xi}$. It is then clear from Eq. (15) that $H_{a}$ admits a global minimum for $D=2$, whenever $X_{s}>Y_{s}$. Also for $D=3, H_{a}$ may exhibit a local minimum under the same condition. The minima of $H_{a}$ are given by the roots of the identity $\delta H_{a} /\left.\delta a\right|_{a=1}=0$, i.e., $X_{s}-D Y_{s} / 2-I_{3}=0$, which is nothing else but the characteristic relation for the ground states, as this can be refound from Eq. (11). This argument points out that the minima of $H$ are reached on the stationary states $\chi$, that constitute stable equilibrium solutions around which any nearby solution can be 
trapped, provided that $X_{s}>Y_{s}$. Note that this inequality is always satisfied by all bound states $\chi$ when $D \geq 2$. For comparison, with the same arguments the free NLS ground states $\left(\Omega_{0}=0\right)$ are found to be unstable and $H_{a}$ has no global minimum for these dimension numbers.

To derive a more rigorous proof for the stability of stationary wavefunctions, we now perturb the latter by means of the following solution

$$
u(r, t)=[\chi(r)+v(r, t)+i w(r, t)] \mathrm{e}^{i \Lambda t},
$$

where $v, w \ll \chi$ are real functions, which we assume to be radially-symmetric. Inserting (16) into Eq. (2), linearizing and decomposing the resulting equation into real and imaginary parts then yield

$$
L_{0} w=\partial_{t} v, \quad L_{1} v=-\partial_{t} w
$$

where the differential operators

$$
L_{0}=-\nabla^{2}+\Lambda-\chi^{2}+\frac{\Omega_{0}^{2}}{4} r^{2}, L_{1}=L_{0}-2 \chi^{2} \text {, }
$$

have the properties

$$
\begin{gathered}
L_{0} \chi=0, \quad L_{1} \partial \chi / \partial \Lambda=-\chi \\
L_{1} \vec{\nabla} \chi=-\Omega_{0}^{2} \vec{r} \chi / 2, \quad L_{0} \vec{r} \chi=-2 \vec{\nabla} \chi .
\end{gathered}
$$

with $\vec{\nabla} \chi \equiv(\vec{r} / r) \partial_{r} \chi$. It is interesting to notice that the small-amplitude perturbations described by Eqs. (16) to (18) provide an alternate form of the usual Bogoliubov excitations. Here, $\chi$ is supposed to be the unique, radially-symmetric solitonlike bound state of Eq. (10) with no zeroes. So, as $L_{0} \equiv-\chi^{-1} \nabla\left[\chi^{2} \nabla(1 / \chi)\right]$ is nonnegative and admits the discrete eigenvalue 0 for $\chi$ only, zero is the lowest eigenvalue of $L_{0}$. In addition, since $\chi$ has no node, $\nabla \chi$ possesses a single node. We then apply a basic theorem from spectral theory claiming that if $\psi_{k}$ is an eigenstate for $L_{1}<L_{0}$ such that $L_{1} \psi_{k}=\lambda_{k} \psi_{k}$, and if $\psi_{k}$ has exactly $k$ zeroes, then $\lambda_{k}$ is the $(k+1)$ th eigenvalue ranked as $\lambda_{0}<\lambda_{1}<\ldots<\lambda_{k}$. This theorem holds when the eigenfunctions of $L_{1}$ are $L^{2}$-integrable, which is here ensured since the linear eigenstates of $L_{1}$ decay much faster with Gaussian tails than their free NLS counterparts. For $\Omega_{0}=0, \lambda_{1}$ is equal to zero and, therefore, a unique negative eigenvalue $\lambda_{0}<0$ exists. Let us now observe what happens with a trapping potential $\left(\Omega_{0} \neq 0\right)$. First, $L_{1}$ has certainly a strictly positive eigenvalue since

$$
<\nabla \chi \mid L_{1} \nabla \chi>=\frac{D \Omega_{0}^{2}}{4} N_{s}>0
$$

where $<\mid>$ denotes the standard $L^{2}$ scalar product applied to real functions. Second, it is obvious that the vectors $\vec{\nabla} \chi$ and $-\vec{r} \chi$ are both orthogonal to the ground state $\chi$. A combination of Eq. (20) then leads to

$$
L_{0} L_{1} \vec{\nabla} \chi=\Omega_{0}^{2} \vec{\nabla} \chi
$$

and, thus, $\vec{\nabla} \chi$ is an eigenstate of $L_{0} L_{1}<L_{0}^{2}$ with positive eigenvalue. For $L_{0}$ being positive definite, this property indicates that $\lambda_{1}>0$. The lowest eigenvalue $\lambda_{0}$ of $L_{1}$ is necessarily negative, although larger than for $\Omega_{0}=0$, because $<\eta \mid L_{1} \eta>$ attains negative values with, e.g., $\eta=\chi$. Moreover, $L_{1}$ has exactly one negative eigenvalue in its spectrum [17]. These technical conditions being fulfilled, we look for perturbations growing as $v, w \sim \mathrm{e}^{\Gamma t}$ with growth rate $\Gamma$ and determine the condition assuring the stability of ground states by maximizing

$$
\Gamma^{2}=-\frac{\langle v| L_{1} v>}{\langle v| L_{0}^{-1} v>}
$$

in the subspace of functions $v$ orthogonal to $\chi$. Stability then results from the proof that $\left\langle v \mid L_{1} v\right\rangle$ is positive under the constraint $\langle v \mid \chi\rangle=0$. According to standard procedures 18 , we identify the discrete spectrum of $L_{1}$ by setting $L_{1} v=\bar{\lambda} v+\alpha \chi$, where $\alpha$ is a Lagrange multiplier related to the orthogonality condition $<v \mid \chi>=0$. We construct a complete basis of orthonormalized eigenfunctions as $\left|v>=\sum_{n} C_{n}\right| \psi_{n}>$, such that $L_{1}\left|\psi_{n}>=\lambda_{n}\right| \psi_{n}>$, and combine these relations to get

$$
G(\bar{\lambda})=\sum_{n} \frac{<\chi\left|\psi_{n}><\psi_{n}\right| \chi>}{\lambda_{n}-\bar{\lambda}}=0 .
$$

In (24), the function $G(\bar{\lambda})$ monotonously increases from $-\infty$ to $+\infty$ in the range $] \lambda_{0}, \lambda_{1}\left[\right.$. With $\lambda_{0}<0$ and $\lambda_{1}>$ 0 , stability follows from the sign of $G(0) \equiv<\chi \mid L_{1}^{-1} \chi>$, which must be negative in order to ensure $\bar{\lambda}>0$. As $G(0)$ is defined by $G(0) \equiv-<\chi \mid \partial \chi / \partial \Lambda>$, we conclude that a sufficient condition for stability is given by

$$
\frac{d N_{s}}{d \Lambda}>0
$$

This condition guarantees the orbital stability of the stationary condensate. By "orbital" stability, it is meant that, modulo the elementary symmetries of Eq. (2), the shape of a BEC soliton is preserved by perturbations having no growing modes, when they act on any solution staying nearby the ground state orbit [17. Conversely, $d N_{s} / d \Lambda<0$ is sufficient for any bound state of Eq.(2) to be unstable. If we moreover admit that $d N_{s} / d \Lambda=0$ also leads to instability, condition (25) is not only sufficient, but also necessary for the stability of ground states.

\section{NUMERICAL RESULTS}

Identified through numerical integrations of Eq. (10), some radially-symmetric, bell-shaped solutions $\chi$ are presented in Figure 1 for different parameters $\Lambda$ and $\Omega_{0}=2$. 
Two-dimensional and three-dimensional ground states are illustrated in Fig. 1(a) and Fig. 1(b), respectively.
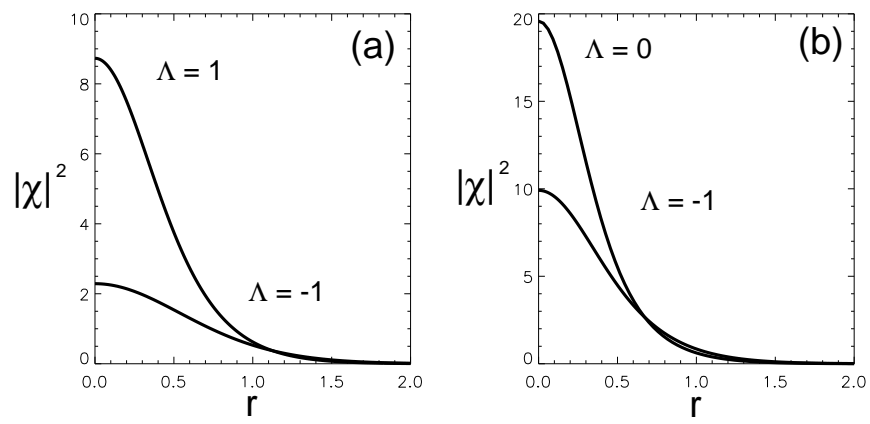

FIG. 1. Examples of radially-symmetric, localized stationary condensates with different $\Lambda$ and $\Omega_{0}=2$, at both dimensions $D=2(\mathrm{a})$ and $D=3(\mathrm{~b})$.

The dependence $N_{s}$ versus $\Lambda$ is shown in Figure 2 for condensates defined at dimension numbers two [Fig. $2(\mathrm{a})]$ and three [Fig. 2(b)], with $\Omega_{0}=2$. Dotted lines indicate the variations of the ground-state particle number $N_{s}$ with respect to $\Lambda$ for the free NLS equation $\left(\Omega_{0}=0\right)$, namely, $N_{s}=11.68$ in $2 \mathrm{D}$ and $N_{s}=18.94 / \sqrt{\Lambda}$ in $3 \mathrm{D}$ [15]. Note that $N_{s}$ for $\Omega_{0} \neq 0$ always lies below these free particle numbers. In the limit $\Lambda \rightarrow+\infty$, the solution $\chi$ behaves as free NLS solitons, which are all unstable with $d N_{s} / d \Lambda \leq 0$. In contrast, as can be seen from this set of figures, trapped ground states are stable for every $\Lambda$ with moderate values when $D=2$, whereas for $D=3$, the criterion (25) predicts their stability for negative $\Lambda$ only, i.e., $\Lambda<\Lambda_{\mathrm{cr}}$, where $\Lambda_{\mathrm{cr}} \simeq-0.72$ corresponds to the maximum number $N_{s}^{\max } \simeq 14.45$.
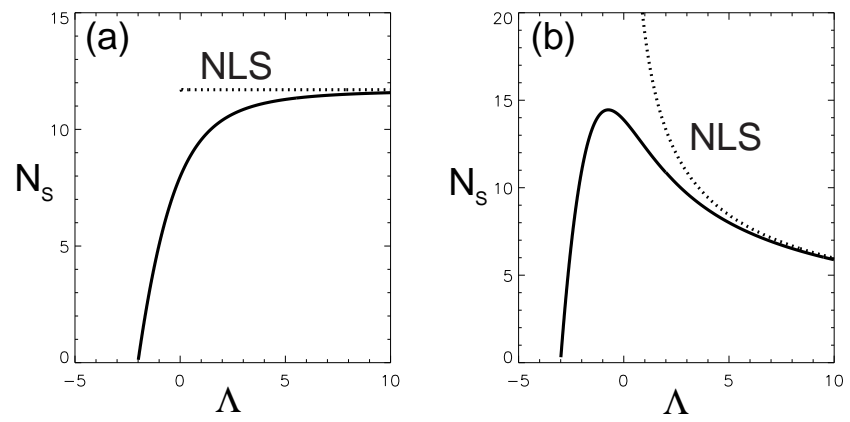

FIG. 2. $N_{s}$ vs. $\Lambda$ for the ground states of Eq. (2). Solid curves refer to $2 \mathrm{D}$ (a) and $3 \mathrm{D}$ (b) confined ground states. Dotted lines indicate their free NLS limits.

The difference between "free" and trapped solutions is that the discrete spectrum of $L_{1}=L_{1}^{\Omega_{0}=0}+\Omega_{0}^{2} r^{2} / 4$ is here shifted towards the range of positive eigenvalues. Thereby, stability of ground states seems "reinforced" by the parabolic trap, compared with the case $\Omega_{0}=0$. Such a result cannot be obtained for a detrapping potential $\left(\Omega_{0}^{2} \rightarrow-\Omega_{0}^{2}\right)$, because the linear eigenstates of $L_{1}$ are not $L^{2}$-integrable in this configuration. Even if the above procedure could be applied in that case, Eq.(21) furthermore suggests that at least two negative eigenvalues would appear in the discrete spectrum of $L_{1}$, from which instability surely follows.

Finally, we have plotted in Fig. 3 the temporal evolutions of solutions to Eq. (2) with a parabolic trap for various initial data. In the $2 \mathrm{D}$ case, Fig. 3(a) shows $|u(0, t)|^{2}$ for initial conditions defined by the ground states $\chi$ with $\Lambda=1$ and $\Lambda=5$ for $\Omega_{0}=2$, and with $\Lambda=1$ for $\Omega_{0}=0$. In the $3 \mathrm{D}$ case, Fig. $3(\mathrm{~b})$ represents similar evolutions from ground states having $\Lambda=-2,-1,0$, among which the solution initiated with $\Lambda=0$ inexorably collapses. Dots show the same initial data undergoing smallamplitude periodic perturbations. From those, we can observe the robustness of the $2 \mathrm{D}$ and $3 \mathrm{D}$ ground states of the GP equation, for which both inequalities (14) and (25) are always verified. Note that perturbations do not affect the ground state orbits, as long as $\Lambda$ is far below the critical values beyond which stationary solutions must become unstable, i.e., $\Lambda \gg 5$ for $D=2$ and $\Lambda>-0.72$ for $D=3$. Stability is lost as $\Lambda$ attains large positive values, in accordance with the criterion (25).
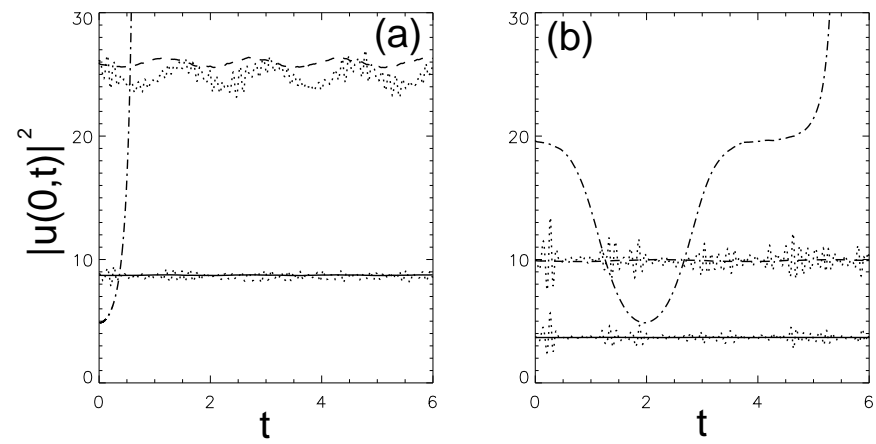

FIG. 3. Temporal evolution of $|u(0, t)|^{2}$ for different initial data with $N=N_{s}$. (a) $D=2, \Lambda=1$ (solid curve), $\Lambda=5$ (dashed) for $\Omega_{0}=2$, and $\Lambda=1$ for $\Omega_{0}=0$ (dash-dotted). This datum promotes a finite-time collapse at $t_{c}=\pi / 2 \Omega_{0}$. (b) $D=3, \Lambda=-2$ (solid), $\Lambda=-1$ (dashed), and $\Lambda=0$ (dash-dotted) for $\Omega_{0}=2$. The dots illustrate the action of perturbations introduced at $t=0$ around the ground states.

\section{CONCLUSION}

We have discriminated the different regions of collapse/no-collapse for the non-stationary solutions of Eq. (2) and determined the exact conditions under which stationary localized states of this equation can exist. We have also demonstrated that the stationary ground states lie in the region $H>H_{\mathrm{cr}}>0$, where collapse is definitively absent in $2 \mathrm{D}$ and can be avoided in $3 \mathrm{D}$, and that they are stable provided $d N_{s} / d \Lambda$ is positive. This result displays evidence that a parabolic trap makes the 
multi-dimensional, stationary solitary modes of the GP equation stable for a wide class of parameters accessible in BEC physics.

To conclude, the criterion (25) allows for determining straightforwardly the stability of condensates from their particle numbers only, which should be useful in current experiments.

[1] M.H. Anderson, J.R. Ensher, M.R. Mattews, C.E. Wieman, and E.A. Cornell, Science 269, 198 (1995); K.B. Davis, M.O. Mewes, M.R. Andrews, N.J. Van Druten, D.S. Durfee, D.M. Kurn, and W. Ketterle, Phys. Rev. Lett. 75, 3969 (1995); see also for review: F. Dalfovo, S. Giorgini, L.P. Pitaevskii, and S. Stringari, Rev. Mod. Phys. 71, 463 (1999).

[2] C.C. Bradley, C.A. Sackett, J.J. Tollet, and R.G. Hulet, Phys. Rev. Lett. 75, 1687 (1995); C.C. Bradley, C.A. Sackett, and R.G. Hulet, 78, 985 (1997); C.A. Sackett, C.C. Bradley, M. Welling, and R.G. Hulet, Appl. Phys. B 65, 433 (1997); C.A. Sackett, H.T.C. Stoof, and R.G. Hulet, Phys. Rev. Lett. 80, 2031 (1998); Yu. Kagan, A.E. Muryshev, and G.V. Shlyapnikov, Phys. Rev. Lett. 81, 933 (1998).

[3] S. Inouye, M.R. Andrews, J. Stenger, H.J. Miesner, D.M. Stamper-Kurn, and W. Ketterle, Nature 392, 151 (1998).

[4] L. Bergé, Phys. Rep. 303, 259 (1998).
[5] L.P. Pitaevskii, Phys. Lett. A 221, 14 (1996).

[6] K. Watanabe, T. Mukai and T. Mukai, Phys. Rev. A 55, 3639 (1997).

[7] T. Tsurumi and M. Wadati, J. Phys. Soc. Jpn. 66, 3035 (1997); ibid. 68, 1531 (1999); M. Wadati and T. Tsurumi, Phys. Lett. A 247, 287 (1998).

[8] C.A. Sackett, J.M. Gerton, M. Welling, and R.G. Hulet, Phys. Rev. Lett. 82, 876 (1999).

[9] C. Huepe, S. Métens, G. Dewel, P. Borckmans, and M.E. Brachet, Phys. Rev. Lett. 82, 1616 (1999).

[10] H. Michinel, V.M. Pérez-García, and R. de la Fuente Phys. Rev. A 60, 1513 (1999).

[11] H. Gauck, M. Hartl, D. Schneble, H. Schnitzler, T. Pfau, and J. Mlynek, Phys. Rev. Lett. 81, 5298 (1998); A.I. Safonov, S.A. Vasilyev, I.S. Yasnikov, I.I. Lukashevich, and S. Jaakkola, Phys. Rev. Lett. 81, 4545 (1998).

[12] S.K. Turitsyn, Phys. Rev. E 56, R3784 (1997).

[13] L. Bergé, Phys. Plasmas 4, 1227 (1997).

[14] M.I. Weinstein, Commun. Math. Phys. 87, 567 (1983).

[15] E.A. Kuznetsov, J. Juul Rasmussen, K. Rypdal, and S.K. Turitsyn, Physica D 87, 273 (1995).

[16] Yu.B. Gaididei, K.Ø. Rasmussen, and P.L. Christiansen, Phys. Rev. E 52, 2951 (1995).

[17] H.A. Rose and M.I. Weinstein, Physica D 30, 207 (1988).

[18] N.G. Vakhitov and A.A. Kolokolov, Izv. Vuz. Radiofiz. 16, 1020 (1973) [Radiophys. and Quantum Electronics 16, 783 (1975)].

[19] A.A. Kolokolov, Izv. Vuz. Radiofiz. 17, 1332 (1974) [Radiophys. and Quantum Electronics 17, 1016 (1976)].

[20] E.A. Kuznetsov, A.M. Rubenchik and V.E. Zakharov, Phys. Rep. 142, 103 (1986). 